\title{
Les noms sociaux en fulfulde
}

Essai de description de la construction des noms sociaux chez les Peuls Social Names in Fula: An Attempt to Describe the Construction of Social Names among the Fulani

\section{Salamatou Sow}

\section{OpenEdition}

\section{Journals}

Édition électronique

URL : https://journals.openedition.org/etudesafricaines/109

DOI : 10.4000/etudesafricaines.109

ISSN : 1777-5353

Éditeur

Éditions de l'EHESS

\section{Édition imprimée}

Date de publication : 1 janvier 2001

Pagination : 557-564

ISBN : 978-2-7132-1394-6

ISSN : 0008-0055

Référence électronique

Salamatou Sow, «Les noms sociaux en fulfulde », Cahiers d'études africaines [En ligne], 163-164 | 2001, mis en ligne le 21 novembre 2013, consulté le 21 septembre 2021. URL : http://

journals.openedition.org/etudesafricaines/109; DOI : https://doi.org/10.4000/etudesafricaines.109 


\section{Salamatou Sow}

\section{Les noms sociaux en fulfulde \\ Essai de description de la construction des noms sociaux chez les Peuls}

Nous appelons noms sociaux ceux par lesquels les Ful'be se désignent pour se distinguer les uns des autres en tant que membres d'une même communauté. Nous savons que les identités peules ont évolué d'abord à travers une identité basée sur le pastoralisme et centrée sur la vache, ensuite à partir de l'islam et des conquêtes territoriales qui ont donné un autre type d'identité centrée sur les territoires ou autour d'une action qui a caractérisé historiquement le groupe, et enfin la colonisation qui a introduit une identité plus large à travers l'ensemble français et dans les États-nations actuels. Comme l'affirment les Ful'be dans leur sagesse: Nde ne'd'do maayaay fuu tagidaaka (Tant que l'homme n'est pas mort, il n'a pas fini d'être créé). L'être humain, tout comme la vie, est une entreprise dynamique.

La société peule n'ayant pas évolué au même rythme, nous trouvons aussi bien des Peuls dévoués encore à l'élevage et à la vache appelés « Peuls de la vache » ("Ful'be na'i»), que ceux attachés à l'islam et à l'instruction coranique désignés comme «Peuls du Livre» ( $F u l$ 'be diina»), ou ceux qui tiennent le pouvoir dans les grands territoires considérés comme «Peuls du tambour» ("Ful'be tube»). Parmi ces Peuls vivent ceux de la cité moderne qui sont fonctionnaires de l'État, commerçants, etc., dont les activités sont ancrées dans la vie moderne.

Nous essaierons de décrire dans cet article le profil du Peul actuel avec ses identités multiples et diverses ainsi qu'à travers les mots avec lesquels il construit ces identités.

Nous présenterons dans une première partie les identités claniques et lignagères, ensuite les identités spatiales ou territoriales, et enfin les nouvelles identités nationales pour dégager la nouvelle dynamique identitaire dans cette époque de démocratie et de globalisation.

\section{Les Peuls de la vache}

L'organisation clanique primordiale

À l'origine, les Ful'be se reconnaissent à travers quatre grands clans qui sont en harmonie d'une part avec les éléments de la nature, le feu, l'air, 
la terre et l'eau; et d'autre part avec les robes des bovidés, jaune, rouge, blanc, noir (Ba \& Dierterlen 1961 : 51). Les pasteurs, vivant au rythme des vaches et de leurs besoins, ont une connaissance aiguë de la nature et de son cycle : les mouvements du soleil, de la lune, des étoiles et le cycle des saisons. Du fait de cette vie dans la nature, les Peuls ont construit une identité qui les place sous le toit du ciel dans la «maison du monde » («suudu duuniya »), dont les repères demeurent les points cardinaux et les astres.

\section{Les clans : une identification originelle}

Les quatre clans primordiaux sont: Jal, Jallo (Dial, Diallo), Ba, Bari, Soh (Sow). À chaque clan est associée une direction, un élément de la nature, une robe de bovidé auquel il est identifié. Ainsi :

— Jal ou Jallo est associé à l'est, au soleil levant, avec le feu comme symbole et la vache jaune oole comme alliée ;

- Ba est configuré à l'ouest, au soleil couchant, symbolisé par l'air et associé à la vache rouge woodeeye ;

- Bari est placé au nord, avec la terre comme symbole et la vache blanche raneeye comme alliée;

- Soh a sa place au sud, avec l'eau comme symbole et la vache noire baleeye comme alliée.

\begin{tabular}{|cc|}
\hline Ouest (air, Ba, rouge) & Nord (terre, Bari, blanche) \\
Est (feu, Jal, Jallo, jaune) \\
Sud (eau, Soh, noire)
\end{tabular}

Cette construction de l'identité première des Ful'be doit être comprise dans le contexte de son «invention » cosmique : l'homme en harmonie avec la nature à partir de laquelle il trouve son activité (élevage) et sa raison de vivre (la vache comme offrande divine). On parle de ce sentiment de peuple élu pour servir la vache sacrée, parce que l'offrande divine conditionnera fortement l'identité des Ful'be na'i.

Un des traits les plus pertinents de cette division clanique réside dans la nécessité de placer fortement l'identité de l'individu dans son clan en rapport avec le monde plutôt que dans sa famille restreinte: l'individu appartient a son clan avant d'appartenir à sa famille.

Ces noms claniques ont encore leur importance de nos jours. Ils sont très vivants dans l'ouest du monde peul (Guinée-Conakry, Guinée-Bissau, Gambie, Sénégal, Mauritanie, Mali, Burkina Faso et l'ouest du Niger), mais ne sont plus utilisés à partir de l'est du Niger et dans toute la partie orientale du monde peul (est du Niger, nord du Nigeria, nord Cameroun, Tchad, République Centrafricaine Soudan, etc.). Ils sont appelés jettooje (pl.) yettoore ou yettoode (sg) qui vient de yetta « remercier, louer ». C'est un nom 
honorifique par lequel on rentre en contact avec un individu et dont le rappel permet de relier l'individu à son origine, à son clan, à travers des actes solennels comme la salutation, le remerciement ou à travers la parole du griot. Ces noms du temps de l'élevage exclusif appartenaient aux pasteurs libres.

La première chose que les gens de ces régions habitués au yettoode vous demande quand vous les rencontrez la première fois, c'est d'abord votre yettoode avant votre prénom, parce que ce dernier permet de vous situer dans un ensemble et de louer, à travers vous, l'ensemble auquel vous appartenez.

Nous pouvons remarquer que ces noms sont formés majoritairement d'une seule syllabe, ce qui n'est pas conforme à la structure des nominaux en fulfulde. Les noms propres peuls authentiques correspondant aux prénoms sont tous formés de deux syllabes : penndo (prénom de la $3^{\mathrm{e}}$ fille), yero (prénom du $3^{\mathrm{e}}$ garçon), etc.

La structure du constituant nominal en fulfulde est :

- $\mathrm{CN}$ = base nominale (bn), qui peut être issue d'une racine nominale, d'une racine verbale ou d'une racine adjectivale suivie facultativement d'un affixe + obligatoirement une modalité nominale $(\mathrm{mn})$.

- $\mathrm{CN}=$ bn $(\mathrm{Vn}, \mathrm{Vv}, \mathrm{Va})+($ affix. $)+$ modalité nominale.

Dans la classification des noms en catégorie + humain/- humain, le fulfulde laisse sans doute une place à une catégorie non classée qui serait peut être celle des Dieux. Nous pouvons dans ce cas mentionner ce que notent Diop (1979, t. 2 : 61) et Matthieu (1988: 38) qui postulent que ces noms seraient d'origine égyptienne et renverraient à des noms de divinités qui symboliseraient chacun un élément naturel (feu, air, terre, eau).

Notons qu'entre ces clans existe une parenté à plaisanterie dans les liens croisés, Jallo avec Ba, Bari avec Soh. Les alliances matrimoniales se liaient aussi dans cette parenté à plaisanterie. Ces noms ont des variantes à travers le monde peul :

- Jal, jallo Ka, Kan ou Kane

- Ba Mbaalo, Balde

- Bari Sangare, ils sont aussi appelés Moodibaa'be (les lettrés)

- Soh Sidibe, Dikko, Soonde.

En marge de cette classification cohérente dans sa représentation, existe d'autres jettooje, réservés aux Toro'b'be, une autre fraction des Peuls non identifiés comme pasteurs mais plus sédentaires. Ils ont comme noms claniques les plus répandus: Ly, Sy et Tal.

Morphologiquement, ce sont des monosyllabes comme les précédents. Les Toro'b’be entretiennent également une parenté à plaisanterie avec les 
Soh. Si l'origine égyptienne des Peuls est admise, les Toro'b'be seraientils une fraction, plus sédentaire donc plus urbaine, des Peuls qui aurait conservé des liens avec le clan qui détenait les secrets initiatiques?

Deux Toro'b'be ont marqué l'histoire des Peuls : il s'agit de Ousmane Dan Fodio et de El Hadj Oumar Tall. Ce sont deux chefs religieux, organisateurs de vastes ensembles islamiques. Ils se sont distingués dans l'érudition et dans l'organisation politique. Les Toro'b'be, qui partagent la même langue et la même culture linguistique que les Peuls, sont plus marqués par la cité et ses pratiques : agriculture, instruction, commerce, etc.

D'autres populations africaines, comme les Mandingues, les Wolofs, les Serer, etc., ont aussi des noms claniques. On peut reconnaître les membres de ces communautés plus urbaines à partir de leurs noms claniques : nobles, griots, forgerons, bijoutiers, etc. Ceci marque une différence avec le système peul construit sur le pastoralisme et qui n'intègre pas les corporations qui appartiennent aux communautés plus spécifiquement sédentaires et urbaines.

Mais de nos jours tous les Peuls ne se reconnaissent pas dans ces grands clans primordiaux. Les migrations successives liées aux pâturages et les aléas climatiques ont dispersé les Peuls dans tout le Sahel et la savane de l'Afrique au sud du Sahara. Ils s'identifient aussi dans des lignages plus restreints à travers lesquels les groupes essayent de préserver leurs particularités.

\section{Les lignages: le lien aux ancêtres}

C'est une classification importante, surtout dans les groupes restés plus nomades où l'organisation est plus restreinte autour d'un ancêtre réel ou «présomptif».

On trouve une multitude de fractions lignagères chez les Wo'daa'be, qui sont restés nomades. Ils sont moins influencés par l'islam et la colonisation. Elles se forment avec:

— 'bii <biy- «enfant de », l'équivalent du Ibn de l'arabe + le nom de l'ancêtre auquel s'identifie le groupe + le morphème nominal marque du collectif «'en» :

'Bii Hamma'en

// enfants/Hamma-collectif//

« descendants de Hamma? »

'Bii Korony'en

«descendants de Korony »

'Bii Gaa'en

«descendants de Gaa»

- l'activité qui caractérise le groupe :

Moodibaa'be «les marabouts» se donnent aux Peuls du clan Bari

Feroo'be «les migrants» se donnent aux Peuls du clan Soh 
- le type de vaches élevées :

Jallinko'en, éleveurs de vaches appelées jalliiji

Bororo'be, éleveurs de vaches bororo

\section{Les Peuls du Livre et des tambours : le territoire comme repère}

Les repères spatiaux porteurs d'identité correspondent:

- aux régions géographiquement ou historiquement importantes ;

- aux terres d'exil, royaumes, gros villages (Fuuta, Maasina, Haayre, Liptaaku, Dallol, Adamaawa, etc.);

- aux repères géographiques : est (fuunaange), ouest (hiirnaange), nord (so'b'birre-woyla), sud (hoore-hu'do, hoor'doore, fommbina).

L'identité du Peul a évolué de la brousse vers la cité à travers l'Islam et les conquêtes qui ont fortifié des positions géographiques propices à l'élevage et abritant des grandes cités peules. Dans ce cas, les noms sont construits à partir de la racine du nom de la région auquel on ajoute le suffixe -anke au singulier et -ankoo'be au pluriel, ou la dernière syllabe du nom redoublée + -jo au singulier et + -'be au pluriel

$\begin{array}{lll}\text { Fuuta } & \text { Fuutanke } & \text { Futankoo'be } \\ \text { Maasina } & \text { Massinanke } & \text { Maasinankoo'be } \\ \text { Adamaawa } & \text { Adamaawanke } & \text { Adamaawankoo'be } \\ \text { Haayre } & \text { Haayranke } & \text { Haayrankoo'be } \\ \text { Dallol } & \text { Dalloojo } & \text { Dalloo'be } \\ \text { Liptaaku } & \text { Liptaakuujo } & \text { Liptaakuu'be }\end{array}$

Ces noms renvoient, au sens large, à tous les habitants de ces régions, et, au sens restreint, aux clans fondateurs de la région.

\section{Les Peuls des États : bi'b'be leydi (fils de la nation)}

L'identité territoriale a créé la notion de 'biiwuraagu (enfant de la maison, $\mathrm{du}$ village ou du royaume). Cette appellation évoluera en 'bii-leydi (enfant du pays, citoyen).

Nom du pays $+-(\mathrm{v})$ nke au singulier et $+-(\mathrm{v})$ nkoo'be au pluriel, quand le nom du pays finit par une voyelle.

Dans ce cas, deux constructions sont possibles :

- Nom du pays + -(v)nke, (v)nkoo'be ou -naajo, -naa'be quand le nom finit par une voyelle :

Égypte Misra Misiranke /Misirankoo'be Égyptien(s)

France Faransi Faransinke/Faransinkoo'be Français 
- Nom du pays $+-\mathbf{n}$-aajo au singulier et $+-\mathbf{n}$-aa'be au pluriel, quand le nom du pays finit par une consonne.

$\begin{array}{llll}\text { Sénégal } & \text { Senegal } & \text { Senegalnaajo/Senegalnaa'be } & \text { Sénégalais } \\ \text { Niger } & \text { Niijer } & \text { Niijernaajo/Niijernaa'be } & \text { Nigérien(s) }\end{array}$

\section{L'identité sociale : une épaisseur à plusieurs couches}

Les identités sociales des Peuls sont multiples : elles sont issues de contacts sociaux multiples qui reflètent d'abord leurs identités d'éleveurs ayant une culture spécifique construite dans la nature " sous le toit du monde », ensuite de sédentaires et de musulmans, et enfin de citadins et de citoyens d'un territoire partageant les mêmes valeurs avec les autres. Les noms claniques sont spécifiques au clan et sont fermés : les individus naissent Jallo ou Bari, etc. Ces noms ne sont pas interchangeables, appartiennent à l'individu et permettent de le situer dans son clan tandis que l'identité liée à un territoire s'étend aux habitants peuls vivant traditionnellement sur l'aire de ce territoire : nous avons aussi bien des Jallo, des Ba, des Bari et des Soh au Massina, qui peuvent être identifiés comme des Masinankoo'be (habitants du Maasina, gens du Massina), ce qui est une appellation plus générale.

Entre les noms claniques et les noms liés à un territoire occupé, les noms des lignages restreints autour d'un ancêtre montrent une identité spécifique qui, comme les noms claniques, situent l'individu dans un lignage qu'il partage avec ses «co-descendants». Les identités claniques, lignagères, territoriales sont les formes courantes d'identification chez les Ful'be. Elles peuvent s'emboîter et participer à l'identification d'un même individu.

Le principe morphosyntaxique de la formation du nom social a aussi évolué d'une formation "religieuse », liée peut-être aux divinités en une syllabe, vers une formation linguistique peule comme dans les noms de lignages et ceux construits à partir des territoires, puis vers une intégration des emprunts nominaux, comme ceux des pays, morphologiquement peulisés par l'adjonction des modalités nominales.

L'histoire humaine est un processus dynamique qui conserve et invente des formes sociales et linguistiques d'adaptation aux autres pour réviser les frontières hors desquelles on envisage l'autre à divers niveaux de différenciations claniques, lignagères ou territoriales dont le nom est porteur.

Université de Niamey

\section{BIBLIOGR APHIE}

Amselle, J.-L. \& M'BокоLо, E.

1999 [1985] Au cæeur de l'ethnie: ethnie, tribalisme et État en Afrique, Paris, La Découverte. 
Azarya, V. \& Eguchi, P. K.

1993 Unity and Diversity of a People : the Search of Fulbe Identity, Senri Ethnological Studies, 35, Osaka, National Museum of Ethnology.

BA, A. H. \& DAget, J.

1984 L'empire peul du Macina, Abidjan-Paris, Nouvelles Éditions africainesEHESS.

BA, A. H. \& Dieterlen, G.

1961 Koumen, textes initiatiques des pasteurs Peul, Paris, Mouton \& Cie.

Botte, R. \& Schmitz, J., eds

1994 «Paradoxes identitaires », Cahiers d'Études africaines, XXXVI (1-3), 133$135: 7$.

Botte, R., Boutrais, J. \& Schmitz, J., eds.

1999 Figures peules, Paris, Kharthala.

Bourgeot, A., ed.

1999 Horizons nomades en Afrique sahélienne, Paris, Karthala.

Bredveld, A. \& De Bruijn, M.

1996 «L'image des Fulbe, analyse critique de la construction du concept de pulaaku », Cahiers d'Études africaines, XXXVI (4), 144 : 791-821.

De Bruijn, M. \& Dijk, H. V.

1997 Peuls et Mandingues: dialectique des constructions identitaires, Paris, Kharthala.

Diop C. A.

1979 Nations nègres et cultures, t. 2, Paris, Présence africaine.

DUPIRE, M.

1996 Peuls nomades. Étude descriptive des Wodaaße du Sahel nigérien, Paris, Karthala.

KILANI, M.

1994 L'invention de l'autre. Essai sur le discours anthropologique, Lausanne, Payot.

LAM, A.

1993 L’origine égyptienne des Peuls, Paris, Présence africaine.

Matthieu, J. M.

1988 Les bergers du soleil, Paris, les Urbanistes.

Riesman, P.

1974 Société et liberté chez, les Peuls Djelgôbé de Haute-Volta, Paris-La Haye, Mouton. 
Seydou, C.

1972 «Une légende peule du Niger occidental : la vache et le Livre », Cahiers des Religions africaines, $12:$ 215-233.

Zuвко, V. G.

1993 «Ethnic and Cultural Characteristics of the Fulbe », in Unity and Diversity of a People: The Search of Fulbe Identity, Senri Ethnological Studies, 35, Osaka : 201-213.

\section{RÉSUMÉ}

Les constructions des identités sociales chez les Peuls reposent sur plusieurs paramètres dont l'activité économique primordiale basée sur la vache et le pastoralisme qui crée une identification sociale plus spécifique autour des clans et des lignages dans la brousse, et l'activité politico-religieuse à travers l'islam et les conquêtes territoriales qui créent une fraternité islamique dans les cités et les régions conquises, en inventant une identification plus large basée sur les territoires. Avec l'histoire coloniale et la création des États-nations, I'identité devient nationale, la citoyenneté prend le pas sur les identités particulières et les noms sociaux manifestent cette dynamique d'une construction identitaire multiple chez les Peuls.

\section{ABSTRACT}

Social Names in Fula: An Attempt to Describe the Construction of Social Names among the Fulani. - Social identities among the Fulani are constructed on several grounds. On the one hand, the primary economic activity cattle-herding creates a social identification with clans and lineages in the bush country. On the other, political and religious activities involving Islam and conquest give shape to an Islamic fraternity in conquered areas and towns, by inventing a broader identification based on the territory. Following colonialism and the formation of nation-states, the sense of identity has become national. Citizenship takes precedence over particularistic identities, and social names provide evidence of the moving construction of multiple identities among the Fulani.

Mots-clés/keywords : Peuls, citoyenneté, clan, constructions, identités, islam, lignage, noms, territoire, vache/Fulani, citizenship, clan, identities, Islam, lineage, names, territory, cattle. 